\title{
Smooth and Controlled Recovery Planning of Disruptions in Rapid Transit Networks
}

\author{
Luis Cadarso, Gábor Maróti, and Ángel Marín
}

\begin{abstract}
This paper studies the disruption management problem of rapid transit rail networks. We consider an integrated model for the recovery of the timetable and the rolling stock schedules. We propose a new approach to deal with large-scale disruptions: we limit the number of simultaneous schedule changes as much as possible, and we control the length of the recovery period, in addition to the traditional objective criteria such as service quality and operational costs. Our new criteria express two goals: the recovery schedules can easily be implemented in practice, and the operations quickly return to the originally planned schedules after the recovery period. We report our computational tests on realistic problem instances of the Spanish rail operator RENFE and demonstrate the potential of this approach by solving different variants of the proposed model.
\end{abstract}

Index Terms-Passengers, railways, recovery, rolling stock, schedule.

\section{INTRODUCTION}

D ESIGNING, planning and operating transport systems, such as railways or public transport systems, is an area where operations research can help substantially.

Railway transportation starts with an extensive planning process involving several combinatorial optimization problems such as line planning, timetabling and rolling stock scheduling.

During the daily operations of a dense passenger railway network, incidents may cause the traffic to deviate from the planned operations. Small-scale incidents, such as a delay of a train by a few minutes, do not require any major intervention from the operator, the delays gradually disappear thanks to the slack of the timetable. In this paper we focus on large-scale incidents, also called disruptions, where significant and nontrivial adjustment of the timetable and the rolling stock schedules is inevitable. In a typical case, a line segment becomes unavailable for a few hours due to causes such as weather

Manuscript received January 8, 2014; revised June 25, 2014, November 26, 2014, and January 27, 2015; accepted February 1, 2015. Date of publication March 19, 2015; date of current version July 31, 2015. This work was supported by the "Ministerio de Economía y Competitividad, Spain," under Grant TRA2011-27791-C03-01. The Associate Editor for this paper was S. C. Wong.

L. Cadarso is with the Department of Signal Theory and Communications, Rey Juan Carlos University, 28943 Madrid, Spain (e-mail: luis.cadarso@ urjc.es).

G. Maróti is with Process Quality and Innovation, Netherlands Railways, 3500 HA, Utrecht The Netherlands, with Utrecht University, 3508 TC Utrecht, The Netherlands, and also with the VU University Amsterdam, $1081 \mathrm{HV}$ Amsterdam, The Netherlands (e-mail: gabor.maroti@ns.nl; g.maroti@vu.nl).

Á. Marín is with the Department of Applied Mathematics for Aerospace Engineering, Technical University of Madrid, 28040 Madrid, Spain (e-mail: angel.marin@upm.es).

Color versions of one or more of the figures in this paper are available online at http://ieeexplore.ieee.org.

Digital Object Identifier 10.1109/TITS.2015.2399975 conditions or mechanical failures. We admit that the distinction between small-scale and large scale incidents is practice driven, it cannot be easily formalized.

In case of a disruption, the operator needs first to compute a new timetable. Then, the rolling stock schedules are modified such that the rolling stock balance between the stations is restored by the end of the day [2]. To produce recovery plans is a complex task since the re-planning has to be done in realtime [15].

Regardless of the cause of a disruption, it has an impact on the railway system. The impact is generally in the form of a change in the system settings, a change in resource availability, or both.

The disruption management process has several objectives. The first goal is to provide the best possible service quality. The second goal aims at easing the rescheduling process by minimizing the differences between the original plan and the recovery plan. Third, the operators often want to quickly return to the original plan once the disruption is over.

This paper studies the integrated timetabling and rolling stock re-scheduling problem in the disruption management of dense passenger railway networks and places a special emphasis on the latter two objectives while providing a high quality service. Any deviation from the undisrupted timetable or from the undisrupted rolling stock assignment is called a schedule change.

We measure the quality of a recovery plan by two metrics: the smoothness and the controllability. Here smoothness refers to the number of simultaneous schedule changes; a smoother recovery plan has fewer schedule changes. Indeed, less smooth plans are often impractical for the operator. Controllability refers to the length of the time period after which the schedules have fully returned to the undisrupted schedule. We call this period the recovery period. The main motivation of controllability is that operators often want to recover before a certain point in time, e.g., before the peak hours.

This paper is organized as follows. Section II provides a literature overview and summarizes the contribution of this paper. Section III describes the problem in detail. Section IV is devoted to the mathematical model. In Section V we present our computational experiments. We draw some conclusions in Section VI.

\section{STATE OF ART}

Reference [14] deals with disruption management in passenger railway transportation. The authors describe the disruption management process and the roles of the different actors involved in it. 
During rail operations, unforeseen events may cause timetable perturbations, which ask for the capability of traffic management systems to reschedule trains and to restore the timetable feasibility ([9], [10]). The dispatchers try to use all available rolling stock to transport as many passengers as possible in the right direction. As a result, the rolling stock units will not finish their daily duties at the location where they were planned to. Reference [2] states that in order to prevent expensive deadheading trips, it is attractive to modify the rolling stock schedules such that the rolling stock is balanced before the night. Reference [19] develops a novel approach in order to reschedule the railway system reducing the current supply along one transportation line in order to reinforce the service of another line (managed by the same operator), which has suffered a disruption. Reference [3] describes a two-stage optimization model for determining robust rolling stock circulations for passenger trains. Here robustness means that the rolling stock circulations can better deal with large disruptions of the railway system. They measure lack of rolling stock capacity based on a given anticipated passenger demand.

All the previous research address the railway re-scheduling problems in a sequential manner. However, sequential solving approach has many drawbacks ([1]). Although practical, the sequential nature of the subproblems leads to sub-optimal plans, with potentially significant economic losses. The airline industry has been a leader in the development of integrated approaches for scheduling and recovering from disruptions. There has been research in the integration of problems such as flight schedule and fleet assignment ([6], [17]), fleet assignment and aircraft routing ([20]), aircraft routing and crew scheduling ([18]), and scheduling and competitive effects ([8], [21]). All these problems were first developed and solved in a sequential fashion. However, integration has outperformed sequential approaches. Reference [22] is among the first ones to deal with the integration of railway timetabling and resource scheduling in disruption management. References [5] and [7] also demonstrate that integrated models provide better solutions for problems within the railway industry. Reference [13] presents a novel weighted train delay based on demand approach for rescheduling railway systems. Reference [16] describes a real-time rolling stock rescheduling model for disruption management which takes dynamic passenger flows into account. The authors describe a heuristic for solving the model with dynamic passenger flows and show that the average delay of the passengers can be reduced significantly.

Rescheduling of airline operations leads to problems comparable to those in railway application. References [11] and [12] combine vehicle scheduling with a dynamic passenger flows. The main difference between the airline and railway settings is that airline passengers are fully controlled by the operator. Our railway application, on the other hand, features passengers who can select their paths through the network.

\section{A. Contributions}

In this paper we present a new approach to deal with largescale disruptions in rapid transit networks. A large-scale disruption usually requires a large number of simultaneous schedule changes in order to recover to the original planning once the disruption has ended. However, it is always difficult to execute a large number of schedule changes at the same time. Another challenge is to control the length of the recovery period: once the disruption has ended the operator wants to recover as fast as possible to the original planning.

The proposed model, which is based on the paper published by [7], is focused on the real-time optimization of train rescheduling (i.e., timetable and rolling stock) and its interactions with the passenger demand.

The main difference between the work in this paper and all the research presented in the literature review is that we explicitly control the recovery length and the number of simultaneous schedule changes to be implemented during the recovery process while recovering the timetable, rolling stock and passenger demand altogether.

The main contributions of this paper are summarized as follows.

- We address the disruption management problem of railway timetable and rolling stock rescheduling with the main focus on providing pragmatic plans for the operator but also accounting for the passenger demand.

- We control the length of the recovery period and limit the number of the simultaneous schedule changes during the recovery from disruptions.

- We carry out computational experiments on realistic study cases of the Spanish rail operator RENFE.

- We demonstrate the potential of this novel approach by solving different variants of the proposed model and showing smoothness and controllability can be achieved at a low cost to both the operator and the passenger demand.

- We perform a multiobjective optimization study in order to study the trade-offs between smoothness and controllability.

We note that we leave certain aspects out of account: our approach allows limited timetable changes, and we model passenger behavior in a heuristic way. We discuss these limitations in Section III-C and E.

\section{PROBLEM DESCRIPTION}

In this section, the recovery problem in rapid transit networks is described. First, disruption characteristics are presented. Then, the railway infrastructure is introduced. Next, we describe train services and shunting in rapid transit networks. Finally, we explain how we treat the passenger demand in case of disturbances.

\section{A. Disruption}

The railway network is composed of stations and arcs; the arcs consist of one or two pairs of rails; the latter case allows simultaneous traffic in both directions.

In this paper, we focus on disruptions that have significant negative disruptive impacts in the transportation system. We make special emphasis on the common type of disruptions when a line segment between two neighboring stations 
becomes fully or partially blocked for a certain time period. Both blockages may have tremendous negative impacts on the system because many services will have to be canceled. But the partial blockage is particularly interesting in that two-way traffic may be scheduled on a single pair of rails. A completely new train schedule is needed because the planned operations are infeasible in the new scenario. However, these negative impacts will not only be limited to the operator but they will also cause passenger demand deviations because some passengers will not be able to realize their travel as it was planned.

Whenever a disruption occurs, the operator will have to recover from it by applying schedule changes such as train delays, train cancellations, and changes of the rolling stock schedules. These types of operations are always difficult to be operated and they are avoided if possible; moreover, the more schedule changes the more human resources are needed.

The two main objectives are the controllability and the smoothness of the recovery plan. These criteria are clearly contradicting: smooth plans need to divide the necessary schedule changes over a longer time period. We are interested in the trade-off between controllability and smoothness. At the same time, we do not want the controllability and smoothness considerations to compromise the operational costs (e.g., the carriage kilometers) and the service quality for passengers.

\section{B. Railway Infrastructure}

The railway network consists of tracks and stations. There exist two different types of stations: passenger and depot stations. The former are stations where passengers get in/off the trains. The latter are the locations where trains are parked or shunted.

We model the infrastructure as a graph with nodes, and with directed arcs. The existing infrastructure between stations is represented by arcs. Between two stations, two different arcs exist, one for each direction of movement.

Rapid transit railway networks usually compete with other transportation modes for passengers (i.e., metro, buses, taxi, etc.). Therefore, we will consider the existence of the Metro network. This Metro network has several stations in common with the rapid transit railway network. However, they are independent, they use different infrastructure and they are operated by different operators. Passengers may find an attractive path using both, the railway and Metro network.

The planning time is discretized into time periods, $t \in T$. Due to the high train frequencies, the duration of one time period is set to one minute. The existing physical network is replicated once for each time period existing in the planning period (e.g., 20 hours).

\section{Timetable}

Timetable information (i.e., departure times and frequencies) is fixed and publicly available for non-disrupted situations. Hence, passengers know when the trains depart and plan their travel accordingly.

Departure times are usually very inflexible because the time slots are negotiated with the infrastructure manager since it may be shared among different operators and lines. However, for disrupted situations there is some freedom to schedule trains with different timetables.

In this research, the timetable consists of three types of train services: the planned train services, emergency services and empty services. Planned train services are the trains scheduled for a normal (undisrupted) situation and they may be canceled due to constraints imposed by some disruption. Emergency services are inserted to the schedule during the disruption in order to alleviate its negative impacts on passengers. Due to the high frequencies, an emergency service may (and mostly does) require the cancellation of a planned service, taking over its timetable slot. Empty services can help satisfy both capacity and rolling stock material availability in depot stations (they do not carry passengers). For every infrastructure element, headway times must be maintained between all the train services which come through.

Our model assumes that the departure and arrival times of a planned service cannot be changed whenever it is not cancelled. This limitation may exclude solutions that would be possible in practice; we need it due to the difficulties that arise when simultaneously handling variable departure and arrival times and the complex rolling stock decisions. We are not aware of any approach that could solve our real-world problem subject to variable event times in reasonable time. In our case study, the effects of this limitation are mitigated by the fact that, during the rush hours, the rapid transport network we study operates close to its maximum capacity.

\section{Rolling Stock and Shunting}

There are self-propelled train units of type $m \in M$; they all have a driver seat at both ends. Train units can be attached to each other to form trains compositions. A composition $c \in$ $C$ of train units is a sequence of elements of $M$ (note that, due to technical limitations, not all sequences of $M$ are valid compositions). Each train unit type has a given capacity; this value includes both seated and standing passengers.

Shunting refers to train operations inside a station. In this paper, shunting is limited to composition changes where the length of an inbound composition is adjusted before its subsequent departure as an outbound train. The adjustments are either disaggregations of a composition into single, or aggregations of a composition from single units. Composition changes, which are only performed in depot stations, enable the operator to have smaller fleet sizes. Composition changes complicate network operation because their processing times are on the same order as the service frequency times. Further complications arise from the necessity of human resources and from the possibility of a mechanical failure during the process. In our case, the operator wishes to restrict the number of composition changes initiated each period to at most one (per depot).

\section{E. Passengers}

Once a disruption has occurred, the network topology changes and passengers will have to use different paths to reach their destination. First, they will have to find a path in 
the modified network, then wait for a train service and finally enter the train if enough capacity is available. We impose a technical assumption that each passenger has a deadline: the last time instant when he is willing to board a train. If he cannot find a seat in any train till his deadline, he is supposed to leave the system, and seek for an alternative mode of transport.

The demand is defined by an origin, a destination and a departure time, that is a passenger group $w \in W$. The size of the passenger group is denoted by $g_{w}$. The network contains different paths $p \in P$ through which the demand will be realized. Each passenger group $w \in W$ will be able to choose a path $p \in P_{w}$, where $P_{w} \subset P$ denotes the set of paths attending $w \in W$. Each path is characterized by its origin, destination and its expected travel time. Due to the fact that several paths with identical origin and destination may exist, passenger groups are assumed to be splittable. Moreover, due to the nature of the transportation system under study, where different modes of transportation exist, different paths coming through different modes of transportation are also taken into account.

Passengers make their trip decisions based on both the timetable and the rolling stock schedule; in addition, rolling stock scheduling needs information about the demand for each trip, which is not available for a disrupted situation. Reference [7] proposes a way to anticipate passenger demand before computing the resource schedules; the anticipated demand is used to guide an integrated optimization model for the timetabling and rolling stock scheduling. This approach is heuristic in that it ignores the dynamic interaction between demand and supplied capacity. This heuristic approach employs a multinomial logit model to represent the passengers' behavior, where the utility of a path is a function of the attributes of the path itself and of the decision-maker.

We will consider the passengers' flows in arcs only, instead of passengers' flows in paths. The per-path demand is transformed into per-arc demand as follows. Let $a$ be an are in the network, and let $\tau$ be a time period in which the demand is to be measured. Then, the per-arc demand is computed by $p f_{a, \tau}=$ $\sum_{w \in W} \sum_{p \in P_{w}} \delta_{a, \tau}^{w, p} \cdot P(p \mid w) \cdot g_{w}, \quad$ where $\quad \delta_{a, \tau}^{w, p} \in\{0,1\}$ expresses whether or not passenger group $w$ using path $p$ is coming through arc $a$ during time period $\tau$ and $P(p \mid w)$ is the probability of choosing a given itinerary $p$ among the set $P_{w}$ by the demand $w$ (see [7]). That is, we assume that each group splits according to the probabilities, and we sum up these split passenger groups on each arc. The values $p f_{a, \tau}$ express the demand for the integrated smooth and controlled re-scheduling model.

The proposed model for the passenger demand is valid as long as each passenger is accommodated in the trains. However, if a passenger cannot take a train (due to insufficient capacity), his/her presence as demand on later trips becomes meaningless. Our optimization model cannot cope with this issue, but [7] justifies this heuristic demand treatment by using an iterative framework where the demand of the next iteration is computed from the optimized timetable of the current iteration. The authors demonstrate that the heuristic approach captures properly the overwhelming majority of the passengers.

\section{INTEGRATED SMOOTH AND CONTROLLED RE-SCHEDULING MODEL}

The Integrated Smooth and Controlled Re-Scheduling Model (ISCREM) aims at computing the timetable and the rolling stock schedule for a disrupted rapid transit network. The ISCREM has its foundations on the models already described in [5] and [7]. These models are in turn extensions of the model described by [4].

The novelty of the current paper lies in Section IV-C2 and $\mathrm{C} 3$ :

- varying penalties for a schedule change based on the time the change is initiated (see the objective function in Section IV-C4);

- the length of the recovery period is controlled (controlled recovery plan) (see Section IV-C2);

- and the number of simultaneous schedule changes is limited (smooth recovery plan) (see Section IV-C3).

The following sections give an overview of the sets and of the decision variables employed in the ISCREM.

A. Sets

- $S$ is the set of stations and $S C \subseteq S$ is the set of depot stations.

- $A$ is the set of arcs.

- $L$ is the set of train services; $L^{p} \subset L$ is the set of planned train services; $L^{\phi} \subset L$ is the set of empty train services; and $L^{e} \subset L$ is the set of emergency train services.

- $T$ is the set of time intervals and $T D_{a} \subseteq T$ is the set of time intervals through which the demand is counted in each $\operatorname{arc} a$.

- $M$ is the train unit type set.

- $C$ is the set of compositions and $C_{m}$ is the set of compositions made of train units type $m$.

- $L_{s, t}$ is the set of train services $\ell$ coming through station $s$ and during the set of time periods $[t, t+h]$, where $h$ is the headway time.

- $L_{a, \tau}$ is the set of train services $\ell$ coming through arc $a$ during time period $\tau$.

\section{B. Variables}

- $x_{\ell, c} \in\{0,1\}$ determines whether composition $c \in C$ is scheduled for service $\ell \in L$. Note that $x_{\ell, c} \in L^{p} \cup L^{e}$ are the only variables that link the timetabling and passengerrelated constraints to the rolling stock constraints.

- $\sigma_{t^{\prime}}^{t} \in \mathbb{Z}^{+}$represents the number of schedule changes that started during time period $t^{\prime}$ and that are being performed during time period $t$.

- $\Gamma_{t} \in\{0,1\}$ controls the length of the recovery period. It takes value 1 if the recovery period is active during time period $t ; 0$, otherwise. The recovery period is defined to be active until the latest time period after which no schedule change starts.

- $y_{\ell} \in\{0,1\}$ determines whether service $\ell \in L^{p}$ is canceled. 
- $y_{s, t}^{c} \in \mathbb{Z}^{+}$gives the number of compositions $c \in C$ at station $s \in S$ at $t \in T$ period.

- $d p_{a, \tau} \in \mathbb{Z}^{+}$is the number of denied passengers due to insufficient capacity in each arc $a, \tau$ defined for $a \in$ $A, \tau \in T D_{a}$.

- $z_{s, t}^{c, c^{\prime}} \in\{0,1\}$ represents whether a composition change starts during $t \in T$ in depot $s \in S C$ from composition $c \in C$ to composition $c^{\prime} \in C$. Recall that at most one composition change can start in each time period.

For the sake of clarity we declared all variables to be integral. We note that the nature of the constraints allows us to relax the integrality of $\sigma_{t^{\prime}}^{t}, d p_{a, \tau}$ and $y_{s, t}^{c}$.

\section{Model Formulation}

Given the above definitions, the model that is used for rescheduling the timetable and the rolling stock for a disrupted rapid transit network can be described as follows:

$$
\min z_{O F}(x, z, y, d p, \sigma, \Gamma)=c(x, z, y, d p)+r(\sigma, \Gamma)
$$

subject to $(2)-(10)$

$$
\begin{aligned}
& \sum_{\ell \in L_{s, t}} \sum_{c \in C} x_{\ell, c} \leq 1 \quad \forall s \in S, t \in T \\
& \sum_{\ell \in L_{a, \tau}} \sum_{c \in C} \operatorname{cap}_{c} x_{\ell, c} \geq p f_{a, \tau}-d p_{a, \tau} \\
& \forall a \in A, \tau \in T D_{a} \\
& \sum_{c \in C} x_{\ell, c}+y_{\ell}=1 \quad \forall \ell \in L^{p} \\
& \sum_{c \in C} x_{\ell, c} \leq 1 \quad \forall \ell \in L^{e} \cup L^{\phi} \\
& y_{s, t-1}^{c}+\sum_{\substack{\ell \in L: \\
\alpha, s, t=1}} x_{\ell, c}+\sum_{c^{\prime} \in C} \gamma_{c^{\prime}, c^{\prime}}^{f} z_{s, t-a d}^{c^{\prime}, c} \\
& =y_{s, t}^{c}+\sum_{\substack{\ell \in L: \\
\alpha \ell, s, t=-1}} x_{\ell, c}+\sum_{c^{\prime} \in C} \gamma_{c, c^{\prime}}^{i} z_{s, t}^{c, c^{\prime}} \\
& \forall s \in S C, t \in T, c \in C \\
& \sum_{s \in S C} \sum_{c \in C_{m}} t u_{c} y_{s, t}^{c}+\sum_{\ell \in L} \sum_{c \in C_{m}} t u_{c} \beta_{\ell, t} x_{\ell, c} \\
& +\sum_{s \in S C} \sum_{t^{\prime} \in T} \sum_{c, c^{\prime} \in C_{m}} \mu_{s, t^{\prime}, t} \gamma_{c, c^{\prime}}^{i} z_{s, t^{\prime}}^{c, c^{\prime}} \leq \chi_{m} \\
& \forall m \in M, t \in T \\
& \sum_{c \in C} t u_{c} y_{s, t}^{c}+\sum_{t^{\prime} \in T} \sum_{c \in C} \mu_{s, t^{\prime}, t} \gamma_{c, c^{\prime}}^{i} z_{s, t^{\prime}}^{c, c^{\prime}} \leq \operatorname{cap}_{s, t} \\
& \forall s \in S C, t \in T \\
& y_{s, t_{i}}^{c}=y_{s, 0}^{c} \quad \forall s \in S C, c \in C \\
& y_{s, t_{f}}^{c}=y_{s, \infty}^{c} \quad \forall s \in S C, c \in C \text {. }
\end{aligned}
$$

The objective function (1) is explained in detail in Section IV-C4 below. Constraints (2) enforce the headway requirements. The constraints state that, during each time interval of length $h$ (the headway time), each station can accommodate at most one departing service at non-zero amount of rolling stock. Constraints (3) link the allocated capacity to the number of passengers $p f_{a, \tau}$. The constraints say that for each arc $a \in A$ and each time interval $\tau \in T D_{a}$, the combined capacity of the trains on the arc during the time interval is enough to accommodate the passenger demand minus the denied passengers. Here $p f_{a, \tau}$ is the passenger demand in each arc $a, \tau$ obtained from the multinomial logit model, while $\operatorname{cap}_{c}$ is the capacity in composition $c$. Constraints (4) state that each planned service is either canceled or it gets exactly one composition. Constraints (5) express that emergency and empty services get at most one composition. Composition conservation constraints (6) ensure the train units' flow balance; the model does not decide the sequence of services that are to be carried out by a train unit, the non-negative inventories make sure that such sequences can indeed be created. The schedule is given by $\alpha_{\ell, s, t}$, which takes the value $1,-1$ or 0 , if train service $\ell$ arrives, leaves or stays in station $s$ at period $t$, respectively. When performing a (dis)aggregation from compositions $c$ to compositions $c^{\prime}, \gamma_{c, c^{\prime}}^{i}$ is the number of needed compositions $c$, and $\gamma_{c, c^{\prime}}^{f}$ is the number of produced compositions $c^{\prime}$. The shunting time ad indicates the time needed to perform an aggregation or disaggregation. Fleet capacity constraints (7) ensure that the number of train units used at time $t \in T$ is limited by the size of the fleet $\chi_{m}$ and depot capacity constraints (8) ensure that the total capacity $\operatorname{cap}_{s, t}$ is not exceeded. $t u_{c}$ is the number of train units in composition $c$. Each train service $\ell$ time duration is given by $\beta_{\ell, t}$, which takes value 1 , if train service $\ell$ is rolling at period $t ; 0$, otherwise. $\mu_{s, t^{\prime}, t}$ gives similar information about performance time of composition changes, which started at $s$ during $t^{\prime}$. Constraints (9) and (10) denote that the inventory during the initial $t_{i}$ and final $t_{f}$ period must be equal to the scheduled one during those time periods $\left(y_{s, 0}^{c}\right.$ and $\left.y_{s, \infty}^{c}\right)$, respectively.

1) Disruption Constraints: In this section we add constraints to the basic rolling stock rescheduling model in order to express the limitations of the disruption. We focus on the common type of distuptions when a line segment between two neighboring stations becomes fully or partially blocked for a certain time period. Due to this disruption's nature we need the following additional variables:

- $\eta_{t}, \theta_{t} \in\{0,1\}$ indicate which of the two riding directions is opened in the line segment between two neighboring stations in period $t \in I T$. If $\eta_{t}=1$ or $\theta_{t}=1$ then one of the riding directions is opened during $t \in I T$ and the opposite direction is closed. IT $\subset T$ is the set of time periods during which the disruption is active

$$
\begin{aligned}
& \sum_{c \in C} x_{\ell, c} \leq \eta_{t} \quad \forall a \in I N O, t \in I T, \\
& \ell \in L_{a}: d t_{d s_{a}}(\ell) \leq t \leq a t_{a s_{a}}(\ell) \\
& \sum_{c \in C} x_{\ell, c} \leq \theta_{t} \quad \forall a \in I S O, t \in I T, \\
& \ell \in L_{a}: d t_{d s_{a}}(\ell) \leq t \leq a t_{a s_{a}}(\ell) \\
& \eta_{t}+\theta_{t} \leq \operatorname{ain}_{t} \quad \forall t \in I T .
\end{aligned}
$$

Constraints (11) and (12) make sure that services can use the disrupted arc only at those time periods when the arc is open 
for their riding direction. $I N O, I S O \subset A$ are the sets of arcs affected by the disruption. The first set contains the arcs with a riding direction which is the opposite one to the riding direction in the second set in an undisturbed situation. $L_{a} \subseteq L$ is the set of train services that use arc $a \in A$. $a t_{a s_{a}}(\ell)$ is the arrival time of service $\ell$ to the arrival station as $a_{a}$ of arc $a$ and $d t_{d s_{a}}(\ell)$ is the departure time of service $\ell$ from the departure station $\mathrm{ds}_{a}$ of arc $a$. Constraints (13) express the infrastructure limitation to one direction at a time $\left(\operatorname{ain}_{t}=1\right)$ or to no traffic at all $\left(\operatorname{ain}_{t}=\right.$ 0 ). The value ain $_{t}=2$ indicates no infrastructure limitation in time period $t$.

2) Recovery Period Constraints: In order to control the recovery period length the following constraints are introduced:

$$
\begin{aligned}
& \Gamma_{t-1} \geq \Gamma_{t} \quad \forall t \in R T \\
& x_{\ell, c}-\hat{x}_{\ell, c} \leq \Gamma_{t} \quad \forall \ell \in L, c \in C, t \in D T_{\ell} \cap R T \\
& \hat{x}_{\ell, c}-x_{\ell, c} \leq \Gamma_{t} \quad \forall \ell \in L, c \in C, t \in D T_{\ell} \cap R T \\
& z_{s, t}^{c, c^{\prime}}-\hat{z}_{s, t}^{c, c^{\prime}} \leq \Gamma_{t} \quad \forall s \in S C, c, c^{\prime} \in C, t \in R T \\
& \hat{z}_{s, t}^{c, c^{\prime}}-z_{s, t}^{c, c^{\prime}} \leq \Gamma_{t} \quad \forall s \in S C, c, c^{\prime} \in C, t \in R T \\
& y_{s, t_{f}}^{c}-\hat{y}_{s, t_{f}}^{c} \leq M_{\infty} \Gamma_{t_{f}} \quad \forall s \in S C, c \in C \\
& \Gamma_{t}=0 \quad \forall t \notin R T .
\end{aligned}
$$

Constraints (14) ensure that the recovery period is active during time period $t$ if and only if it is active during the immediately predecessor time period $t-1$; here, $R T$ is the set of time periods that are allowed by the operator to be within the recovery period. Recall that the recovery period is considered active until the latest time period when a schedule change starts. Constraints (15)-(18) ensure that schedule changes related to a train and a composition change can occur if the recovery period is active; here, $D T_{\ell}$ represents the departure time of train service $\ell . \hat{x}_{\ell, c}, \hat{z}_{s, t^{\prime}}^{c, c^{\prime}}$ and $\hat{y}_{s, t}^{c}$ indicate the rolling stock assignment, the composition changes and the train inventory on a normal day. In essence, these four constraints say that, with a slight abuse of notation, if $\Gamma=0$ then $x=\hat{x}$ and $z=\hat{z}$ hold. Constraints (19) feature a sufficiently large value $M_{\infty}$, and they state that if $\Gamma$ corresponding to the end of the day is zero then each station's end-of-day inventory is at most the originally planned inventory value. Observe that $\sum_{c, s} y_{s, t_{f}}^{c}=\sum_{c, s} \hat{y}_{s, t_{f}}^{c}$. Thus constraints (19) say that $\Gamma_{t_{f}}=0$ implies $y_{s, t_{f}}^{c}=\hat{y}_{s, t_{f}}^{c}$ for each composition set $c$ and for each station $s$. Finally, constraints (20) ensure that the recovery period is not active for all the time periods not contained in $R T$.

3) Schedule Changes Constraints: Finally, the amount of schedule changes during the recovery period is limited by the following constraints:

$$
\begin{aligned}
\sigma_{t^{\prime}}^{t}= & \sum_{\ell \in L_{t^{\prime}}} \sum_{c \in C} \beta_{l, t}\left|x_{\ell, c}-\hat{x}_{\ell, c}\right| \\
& +\sum_{s \in S C} \sum_{c, c^{\prime} \in C} \mu_{s, t^{\prime}, t}\left|z_{s, t^{\prime}}^{c, c^{\prime}}-\hat{z}_{s, t^{\prime}}^{c, c^{\prime}}\right| \\
& +\sum_{s \in S C} \sum_{c \in C} \delta_{t}^{t^{\prime}}\left|y_{s, t}^{c}-\hat{y}_{s, t}^{c}\right| \quad \forall t, t^{\prime} \in R T: t \geq t^{\prime} \\
& \sum_{t^{\prime} \in R T: t \geq t^{\prime}} \sigma_{t^{\prime}}^{t} \leq v \quad \forall t \in R T .
\end{aligned}
$$

Constraints (21) count the number of schedule changes which began during time period $t^{\prime}$ and are being performed during time period $t$. These constraints count schedule changes related to train, emergency and empty services, composition changes and train inventory at depot stations; $L_{t^{\prime}}$ is the subset of train services which depart at $t^{\prime}$ and $\delta_{t}^{t^{\prime}}$ is 1 if $t$ is equal to $t^{\prime}$. Constraints (22) limit the number of schedule changes to be operated during every time period $t$ to a maximum value $v$.

All the terms on the right hand side of constraints (21) are non-linear; however, the binary character of the variables $x_{\ell, c}$ and $z_{s, t^{\prime}}^{c, c^{\prime}}$ admits a straightforward linearization; the last term requires a slightly more complex linearization

$$
\begin{aligned}
\sigma_{t^{\prime}}^{t}= & \sum_{\ell \in L_{t^{\prime}}} \sum_{c \in C: \hat{x}_{\ell, c}=0} \beta_{l, t} x_{\ell, c} \\
& +\sum_{\ell \in L_{t^{\prime}}} \sum_{c \in C: \hat{x}_{\ell, c}=1} \beta_{l, t}\left(1-x_{\ell, c}\right) \\
& +\sum_{s \in S C} \sum_{c, c^{\prime} \in C: \hat{z}_{s, c^{\prime}}^{c, c^{\prime}}=0} \mu_{s, t^{\prime}, t z_{s, t^{\prime}}^{c, c^{\prime}}} \\
& +\sum_{s \in S C} \sum_{c, c^{\prime} \in C: \hat{z}_{s, t^{\prime}}^{c, c^{\prime}}=1} \mu_{s, t^{\prime}, t}\left(1-z_{s, t^{\prime}}^{c, c^{\prime}}\right) \\
& +\sum_{s \in S C} \sum_{c \in C} \delta_{t}^{t^{\prime}} \epsilon_{s, t}^{c} \quad \forall t, t^{\prime} \in R T: t \geq t^{\prime} \\
y_{s, t}^{c}- & \hat{y}_{s, t}^{c} \geq-\epsilon_{s, t}^{c} \quad \forall s \in S C, t \in R T, c \in C \\
y_{s, t}^{c}-\hat{y}_{s, t}^{c} \leq & \forall s \in S C, t \in R T, c \in C
\end{aligned}
$$

where $\epsilon_{s, t}^{c}$ are non-negative variables. Note that whenever $\sigma_{t^{\prime}}^{t}$ has a positive coefficient in the objective, any optimal solution satisfies $\left|y_{s, t}^{c}-\hat{y}_{s, t}^{c}\right|=\epsilon_{s, t}^{c}$.

4) Objective Function: The linear objective function (1) of the ISCREM consists of two parts: the operator and passenger related costs $c(x, z, y, d p)$ and the recovery schedule quality related costs $r(\sigma, \Gamma)$. The operator and passenger related costs penalize the following quantities. (i) Operating costs of planned, empty and emergency services; here $o c_{c}$ is the operating cost per kilometer and $k m_{\ell}$ is the distance in kilometers of service $\ell$. (ii) Composition changes; here $\vartheta_{s, t}$ is the cost of a composition change at depot $s$ in time period $t$. (iii) Cancellation of services; here $c^{2} a c_{\ell}$ is the cancellation cost for service $\ell$. (iv) Denied passengers; here $d p c_{a, \tau}$ is the cost per denied passenger due to insufficient capacity in each arc $a$ during time period $\tau$. That is

$$
\begin{aligned}
c(x, z, y, d p)= & \sum_{\ell \in L} \sum_{c \in C} o c_{c} k m_{\ell} x_{\ell, c}+\sum_{s \in S C} \sum_{t \in T} \sum_{c, c^{\prime} \in C} \vartheta_{s, t} z_{s, t}^{c, c^{\prime}} \\
& +\sum_{\ell \in L^{p}} c a n c_{\ell} y_{\ell}+\sum_{a \in A} \sum_{\tau \in T} d p c_{a, \tau} d p_{a, \tau} .
\end{aligned}
$$

The recovery schedule quality related costs penalize the following quantities. (i) Deviation from the planned schedule; every schedule change has an associated penalty $p_{\zeta}$ which depends on the time period $\zeta$ it starts. (ii) Every time period during which the recovery is active has an associated penalty cost $t_{t}$. That is

$$
r(\sigma, \Gamma)=\sum_{\zeta=t_{i}}^{t_{f}} p_{\zeta} \sum_{\tau=\zeta}^{t_{f}} \sigma_{\zeta}^{\tau}+\sum_{t=t_{i}}^{t_{f}} \operatorname{cost}_{t} \Gamma_{t} .
$$



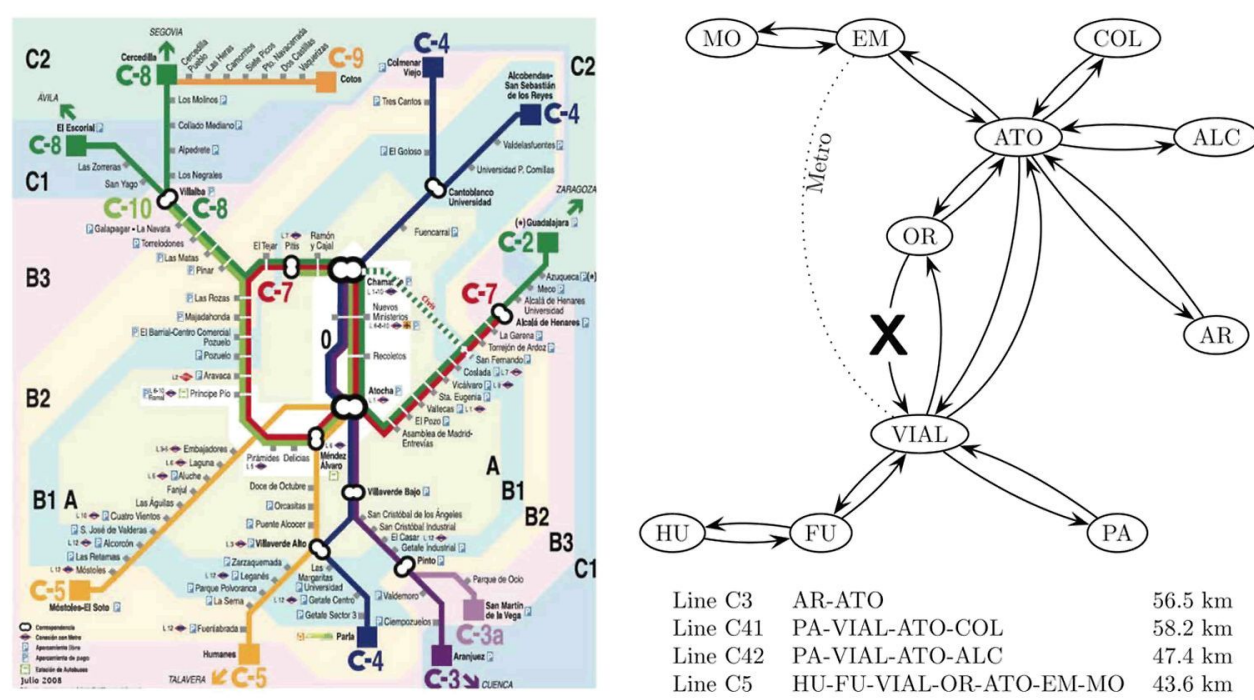

Fig. 1. Left: RENFE's Rapid Transit Network around Madrid. Right: The network topology while the blockage is active; the dashed arrows indicate the independently operated Metro line. A cross indicates the disrupted segment between OR and VIAL.

\section{Computational Experiments}

Our experiments are based on realistic cases drawn from RENFE's network in Madrid for 2008 (on the left in Fig. 1). The whole network is composed of 10 different lines with almost 100 stations, carrying more than one million passengers every day. The network has double tracks on all segments. In this section, we study the disruption that has been already analyzed in [7]; this allows a direct comparison of our results to those of [7]. We report our results on a case study with the failure of a particular line segment during a particular time interval. We did carry out tests on three other cases that concern another line segment, another time interval, or both. The results on the three cases are in line with the conclusions of Section V-C, therefore we omit the details.

We used for our tests a personal computer with an Intel Core 2 Quad CPU at $2.83 \mathrm{GHz}$ and $8 \mathrm{~GB}$ of RAM, running under Windows 7 64-Bit, and we implemented the models in GAMS/Cplex 12.1.

\section{A. Case Description}

This case study concerns a disruption where one of the two tracks between two stations is blocked: trains in different directions must share the remaining track. Also, some trains that were supposed to pass may turn back instead of entering the disrupted segment. The disruption starts at 8:00 A.M. and it lasts 120 minutes.

The disrupted segment is only used by trains belonging to the C5 line. Passengers of line $\mathrm{C} 5$ have multiple traveling options: they can remain in the line $\mathrm{C} 5$ waiting for a direct or indirect train; they also can make use of lines $\mathrm{C} 3, \mathrm{C} 41$, and $\mathrm{C} 42$ as well as of a line of the Metro network. The Metro line is considered because it provides a particularly convenient way to circumvent the blockage. The disruption has no direct effect on the passengers of lines $\mathrm{C} 3, \mathrm{C} 41$, and $\mathrm{C} 42$; they will just stick to their intended paths. We note that the lines $\mathrm{C} 3, \mathrm{C} 41, \mathrm{C} 42$, and $\mathrm{C} 5$ belong to RENFE while the Metro network is run by another operator.
The travel choices of the passengers can be represented by a network restricted to the aforementioned lines only. The restricted network, depicted on the right in Fig. 1, features 46 stations, and about 12000 trips in 760 timetable services. About 530000 passengers use the restricted network, 47000 of which are directly affected by the disruption. The frequency on the $\mathrm{C} 5$ line is rather high: there is a train service every 3 minutes in the peak hours and every 10 minutes in the off-peak hours. Lines C3, C41, and C42 have a slightly lower frequency: trains in the peak hours run every 6 minutes and every 16 minutes in the off-peak hours. The considered lines are served by two train unit types with a capacity of 588 and 757 , respectively. Trains on the Metro line run every 3 minutes and we assume that they have unlimited capacity.

The integrated timetable and rolling stock rescheduling problem instances are solved by two approaches: by the model of [7]; and by the model proposed in this paper (which is an extension of [7]).

The optimization models can make timetabling decisions in Line C5: cancellations of existing services or insertions of emergency services. The model selects emergency services from a pool of potential candidates which depart every minute and go through the non-blocked area. Also, the model decides on the rolling stock allocation of the lines $\mathrm{C} 5, \mathrm{C} 3, \mathrm{C} 41$, and $\mathrm{C} 42$. The undisrupted lines are incorporated in order to adjust the train capacities to the elevated demand figures. We do forbid, though, the cancellation of any of the $\mathrm{C} 3, \mathrm{C} 41$, and $\mathrm{C} 42$ services.

The objective functions arise as a weighted combination of different terms; the relative importance of the criteria expresses different overall managerial goals. In order to compare the two solution approaches, we use a particular weight setting for the objective criteria.

\section{B. Recovery Solutions}

In this section we describe and compare solutions to the integrated optimization problem. The complete ISCREM model (1)-(25) leads to a mixed integer program with about 39000 
TABLE I

RECOVERY SOLUTIONS

\begin{tabular}{ccccccc}
\hline Schedule & TSOC & EMOC & SC & DP & DP-est & ST \\
\hline Non-Controlled-Non-Smooth & 166338.06 & 6984.63 & $5537\left(64^{*}\right)$ & 1839 & 5221 & 206 \\
Controlled-Non-Smooth & 166912.21 & 7782.05 & $1659\left(70^{*}\right)$ & 1914 & 5262 & $278\left(116^{* *}\right)$ \\
Controlled-Smooth & 167208.37 & 7798.58 & $712\left(59^{*}\right)$ & 1998 & 5388 & $614\left(201^{* *}\right)$ \\
Undisrupted & 167937.28 & 5355.87 & - & - & - & 122.5 \\
\hline
\end{tabular}

*Total Number of schedule changes related to services and composition changes. **Computational time for $1 \%$ of relative optimality gap.

discrete variables, about 1390000 continuous variables and about 487000 constraints.

Table I summarizes our results by letting each row represent one of the four schedules. Non-Controlled-Non-Smooth: obtained by solving the disruption without accounting for controllability and smoothness of the recovery. This solution is directly quoted from [7]. Controlled-Non-Smooth: obtained by accounting for recovery period constraints Section IV-C2, but not considering the number of simultaneous schedule changes (constraints (22)). Controlled-Smooth: obtained by solving the ISCREM as described in Section IV. In particular, we do use constraints (22); the upper bound in (22) is defined as $75 \%$ of the number of simultaneous schedule changes in the solution Controlled-Non-Smooth. Undisrupted: refers to the schedule of an undisrupted day; this schedule comes from the optimization model proposed by [4].

Table I shows six characteristics of the solutions. Columns TSOC and EMOC give the total operational costs for passenger train services and empty movements, respectively. Note that the first term in (26) is equal to the sum of TSOC and EMOC. Column SC, the total number schedule changes, is a measure to estimate how easy it is for the operator to implement the recovery plan; SC accounts for train service changes, composition changes and train inventory changes. Column DP gives the number of denied passengers calculated in a post-processing step, while column DP-est is the number of denied passengers as estimated by the optimization model. Finally, column ST gives the solution time in seconds.

We note that the distinction between DP and DP-est is necessary due to the fact that the ISCREM models the denied passengers heuristically: the demand on successive arcs are not linked to each other. Therefore, a denied passenger still shows up in the demand of later arcs. For more details and justifications we refer to [7].

We first notice that the lowest possible DP is that one in the Non-Controlled-Non-Smooth solution; this solution is the least constrained one. The service quality deteriorates slightly as we impose more and more restrictions on the schedules.

The solutions do show clear differences for the ease of the recovery process, measured by the total number of schedule changes (SC). The results conform with our intuition: solutions Controlled-Smooth, Controlled-Non-Smooth and NonControlled-Non-Smooth have increasingly more freedom to change the schedules, and they indeed use this freedom to reach a better service quality. The Controlled-Smooth solution makes $\mathrm{SC}$ dramatically drop while the other values stay more or less the same.

The operational costs (TSOC and EMOC) do not show much variation. As one may expect, the Non-Controlled-Non-
Smooth solution is the best, but the Controlled-Non-Smooth and Controlled-Smooth solutions are reasonably close to it. When comparing to the undisrupted schedule, the recovery schedules have lower costs (TSOC) for train and emergency services. This follows from the fact that some train services are canceled. On the other hand, the recovery schedules have higher empty service costs (EMOC) because more empty services are needed to match capacity requirements and rolling stock resources.

The solution times (ST) range from a few seconds to a few minutes. We notice that the recovery schedules need a higher computational time, among others due to the additional complexity of the timetabling decisions. All in all, the proposed model fits well in the time frame of real life disruption management.

\section{Comparison of Non-Controlled-Non-Smooth and Controlled-Smooth Solutions}

Section V-B gave the high-level statistics of three recovery solutions. Here we take a closer look at them in order to assess their practical value. The assessment is based on the total number of schedule changes and on their temporal distribution. Desirable recovery solutions do not have too many of them, and they are to be limited to a short time interval just after the disruption.

Fig. 2 shows the number of schedule changes (as a function of time) in the Non-Controlled-Non-Smooth, the ControlledNon-Smooth and the Controlled-Smooth solutions. The figure has a curve for each of the solutions. These curves illustrate the difficulty of each of the solutions to be implemented in practice. The disruption of our case study ends at 10:00 AM. The NonControlled-Non-Smooth solution has schedule changes until the end of the day. This is undesirable because the effects of the disruption spread out for the whole day, including the afternoon peak hours. For the Controlled-Non-Smooth solution, the length of the recovery period is at 2:00 PM. Consequently, the downstream effects of the disruption are now controlled. The operations are recovered from the disruption by the time the afternoon peak period starts. Finally, in the ControlledSmooth solution, the maximum number of simultaneous schedule changes is required to decrease by $25 \%$. The additional requirements leads to an even shorter recovery period.

The area under the curves indicates the number of schedule changes weighted by their duration. The area can thus be perceived as an intuitive quality measure of the recovery plans. It confirms our finding that Controlled-Smooth is superior to the other solutions for its controllability and smoothness.

Limiting the recovery efforts (in terms of maximum simultaneous schedule changes and length of recovery period) 


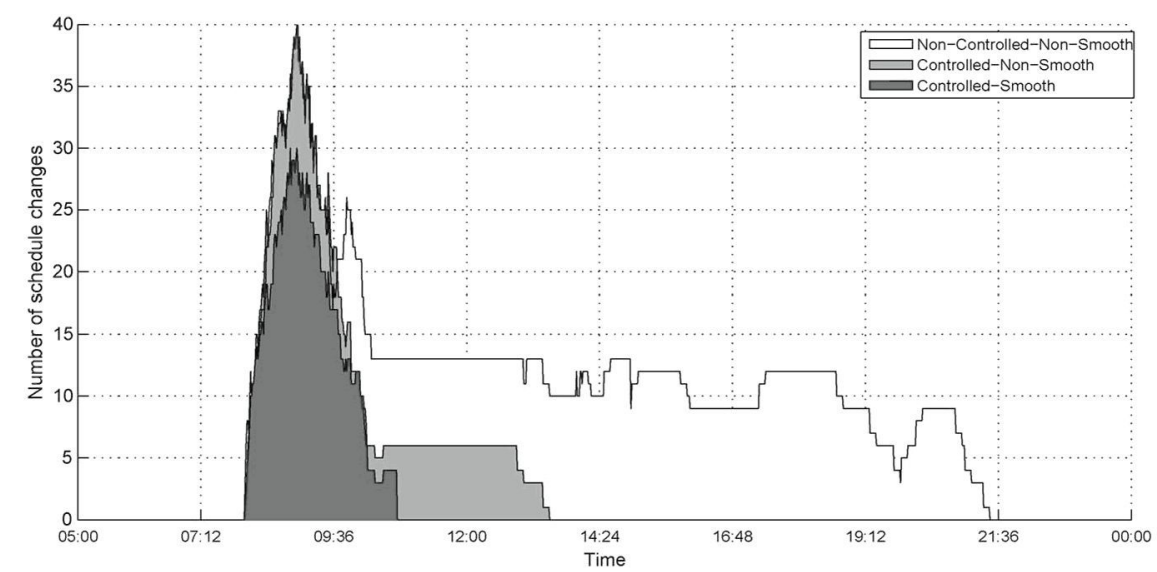

Fig. 2. Number of schedule changes in the Non-Controlled-Non-Smooth, the Controlled-Non-Smooth and the Controlled-Smooth solutions.

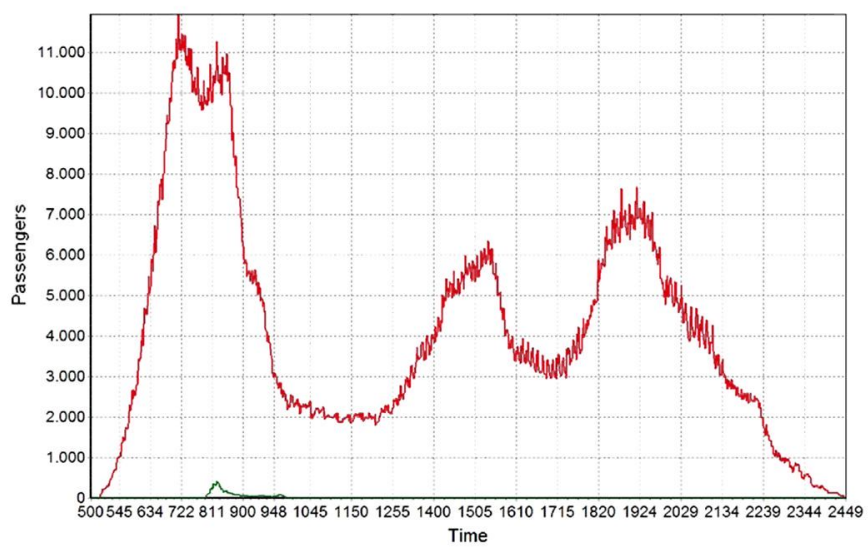

Fig. 3. Passenger demand in the Non-Controlled-Non-Smooth solution: number of requested trips (higher curve) and number of denied trips (lower curve).

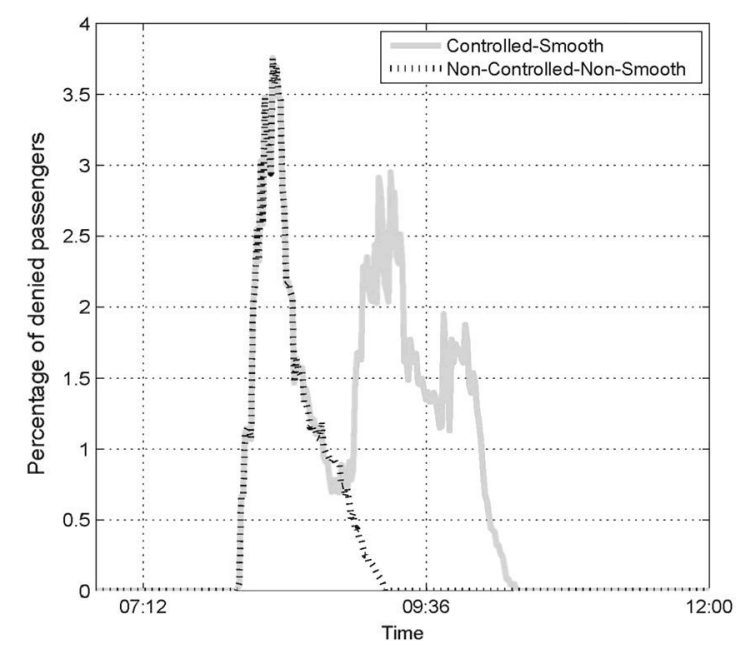

Fig. 4. Percentage of denied passenger demand in the Non-Controlled-NonSmooth solution and Controlled-Smooth solution (b).

\section{Smoothness and Controllability}

Smoothness and controllability of the recovery plan are contradictory objectives: smooth plans need to spread out schedule changes over a longer time period and controlled plans need to compact them. We are interested in the trade-off between controllability and smoothness. Therefore, we propose a new objective function in order to make a bi-objective study as follows:

$$
\min \omega v+(1-\omega) \sum_{t=t_{i}}^{t_{f}} \Gamma_{t}
$$

subject to (2)-(19) and (21)-(25). Here $v$ is a positive variable representing the maximum number of simultaneous schedule changes and $\omega \in[0,1]$. Different values of $\omega$ may yield different Pareto solutions for the new bi-objective problem. Fig. 5 shows the Pareto frontier: the $\mathrm{x}$-axis represents the length of the recovery period in minutes and the $y$-axis the maximum number of simultaneous schedule changes. Obviously, this Pareto frontier does not take care of the traditional criteria, namely the operational costs (e.g., the carriage kilometers) and the service 


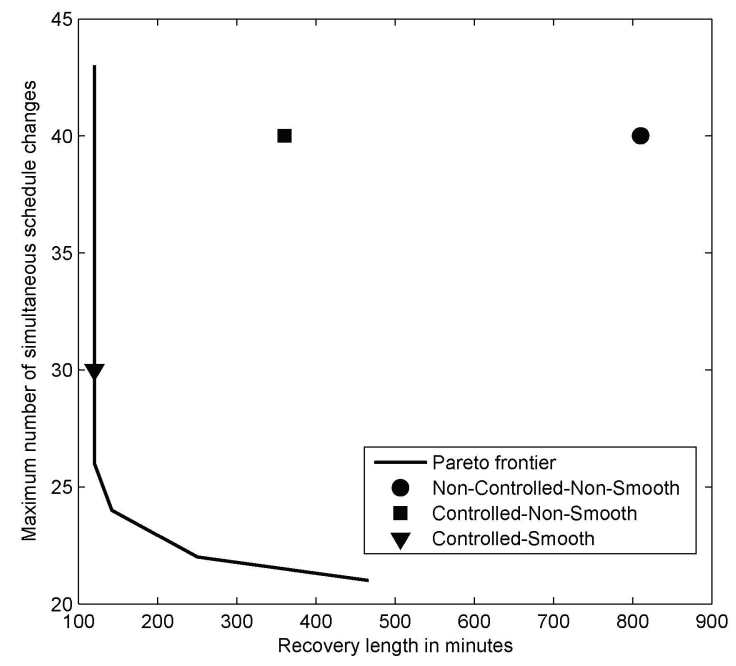

Fig. 5. Pareto frontier.

quality for passengers. Therefore, the Pareto optimal solution may compromise the overall optimal solution given by the model in (1)-(25). However, the objective (28) minimizes the deviation from the undisrupted solution, therefore it takes the traditional criteria implicitly into account. For a comparison, Fig. 5 also plots the solutions Non-Controlled-NonSmooth, Controlled-Non-Smooth and Controlled-Smooth.

The Controlled-Smooth solution turns out to be Pareto optimal; in fact, it features the shortest possible recovery period. There exist solutions with the same the recovery period length and with fewer simultaneous schedule changes; those solutions are more expensive in terms the objective function (1). Nevertheless, the Pareto optimal solutions are very similar for their value in (1): their differ by at most $4.97 \%$ from the objective value of Controlled-Smooth. This result confirms that the tradeoff mainly lies between smoothness and controllability. The operator can select the most preferred Pareto optimal solution without causing any major suboptimality in terms of the objective function (1).

The modified objective (28) is computationally more challenging that our original objective (1). We experienced solution times of up to 17000 seconds, mostly in cases where the number of simultaneous schedule changes is penalized more heavily. In contrast, the Controlled-Smooth solution needed 614 seconds.

\section{E. Dealing With Denied Passengers}

In this section we consider the Controlled-Smooth solution and we investigate the accuracy of our heuristic passenger model. The Non-Controlled-Non-Smooth and Controlled-NonSmooth solutions behave very similarly.

Inaccuracy occurs for denied passengers. Of the 1998 denied passengers, 1314 passengers leave the system because they reached their deadline. These passengers are handled properly by the optimization model. However, the remaining 684 passengers did attempt to board a train but were rejected due to insufficient capacity. Still, our passenger model adds them to the demand on their entire path, even after the rejection. Thus the bogus demand may lead to unnecessary rejections. We give a conservative estimate on the inconvenience of the rejected passengers by assuming that the rejected passengers wait for the earliest available free seat in a train on their chosen path to their destination. Their delay is defined as the difference between their rejection time and the time when a seat does become available for them. It turns out that the rejected passengers suffer an average delay of 12.8 minutes, the values ranging from 1 minute to 37 minutes; $78.9 \%$ of the delays under 20 minutes, 144 passenger have a higher delay.

We conclude that heuristic treatment in our model gives rise to realistic passenger flows with a small fraction of the passengers denied. Moreover, the large majority of these inaccurately modeled passengers can travel with a tolerable delay. The passengers without a reasonable travel option form a fraction whose size is below the daily fluctuation of the demand figures.

\section{CONCLUSION}

In this paper we study the recovery problem of rapid transit networks. When dealing with a disruption, the operator wants to offer a good service quality and to quickly steer the system back to the original plan.

A main contribution with respect the literature is that our approach decides on the recovery length and the number of simultaneous schedule changes to be implemented during the recovery process while recovering the timetable, rolling stock and passenger demand altogether.

Our model balances several system-related and servicerelated objective criteria and puts a particular emphasis on the objective of returning to the original plan as quickly and as easily as possible, i.e., limiting the disruptive negative impacts in terms of schedule changes and recovery time period length but also offering o good quality service to the passenger demand.

The computational tests on realistic instances of RENFE show that our method is able to find solutions with a very good balance between the stated managerial goals. The smooth and controlled variant of the model reduces the number of simultaneous schedule changes and greatly limits the length of the recovery period-without compromising the service quality. In addition, we compare the solutions we found to the Pareto frontier of the bi-objective minimization problem. Discussions with practitioners revealed that the solutions captured all important real-life restrictions, and have a good chance to be implementable in practice.

The computational times amount to a few minutes which is sufficiently close to the needs of real-time decision making. This is a great advantage with respect to the current system of manual re-planning where planners work under great time pressure.

\section{ACKNOWLEDGMENT}

The first author thanks the Railway Applications Section of INFORMS, for awarding this research in the 2013 Student Paper Award. The authors also want to thank the anonymous referees for their helpful comments on the paper. 


\section{REFERENCES}

[1] C. Barnhart, "Airline schedule optimization," in The Global Airline Industry, P. Belobaba, A. Odoni, and C. Barnhart, Eds. Hoboken, NJ, USA: Wiley, 2009.

[2] G. Budai, G. Maróti, R. Dekker, D. Huisman, and L. G. Kroon, "Rescheduling in passenger railways: The rolling stock rebalancing problem," J. Sched., vol. 13, no. 3, pp. 281-297, Jun. 2010.

[3] V. Cacchiani et al., "Railway rolling stock planning: Robustness against large disruptions," Transp. Sci., vol. 46, no. 2, pp. 217-232, May 2012.

[4] L. Cadarso and A. Marín, "Robust rolling stock in rapid transit networks," Comput. Oper. Res., vol. 38, no. 8, pp. 1131-1142, Aug. 2011

[5] L. Cadarso and A. Marín, "Integration of timetable planning and rolling stock in rapid transit networks," Ann. Oper. Res., vol. 199, no. 1, pp. 113-135, Oct. 2012.

[6] L. Cadarso and A. Marín, "Robust passenger oriented timetable and fleet assignment integration in airline planning," J. Air Transp. Manage., vol. 26, pp. 44-49, Jan. 2013.

[7] L. Cadarso, A. Marín, and G. Maróti, "Recovery of disruptions in rapid transit networks," Transp. Res. E, Logist. Transp. Rev., vol. 53, pp. 15-33, Jul. 2013.

[8] L. Cadarso, V. Vaze, C. Barnhart, and A. Marín, "Integrated airline scheduling: Considering competition effects and the entry of the high speed rail," Transp. Sci., Feb. 2015, accepted for publication.

[9] A. D'Ariano, M. Pranzo, and I. A. Hansen, "Conflict resolution and train speed coordination for solving real-time timetable perturbations," IEEE Trans. Intell. Transp. Syst., vol. 8, no. 2, pp. 208-222, Jun. 2007.

[10] A. D'Ariano and M. Pranzo, "An advanced real-time train dispatching system for minimizing the propagation of delays in a dispatching area under severe disturbances," Netw. Spatial Econ., vol. 9, no. 1, pp. 63-84, Mar. 2009

[11] J. Dumas and F. Soumis, "Passenger flow model for airline networks," Transp. Sci., vol. 42, no. 2, pp. 197-207, May 2008.

[12] J. Dumas, F. Aithnard, and F. Soumis, "Improving the objective function of the fleet assignment problem," Transp. Res. B, Methodol., vol. 43, no. 4, pp. 466-475, May 2009.

[13] J. L. Espinosa-Aranda and R. García-Ródenas, "A demand-based weighted train delay approach for rescheduling railway networks in real time," J. Rail Transp. Planning Manage., vol. 3, no. 1/2, pp. 1-13, Feb.-May 2013.

[14] J. Jespersen-Groth et al., "Disruption management in passenger railway transportation," in Robust and Online Large-Scale Optimization, Lecture Notes in Computer Science, vol. 5868, R. Ahuja, R. Möhring, and C. Zaroliagis, Eds. Berlin, Germany: Springer-Verlag, 2009, pp. $399-421$.

[15] L. G. Kroon and D. Huisman, "Algorithmic support for railway disruption management," in Transitions Towards Sustainable Mobility, J. A. E. E. Nunen, P. Huijbregts, P. Rietveld Eds. Berlin, Germany: Springer-Verlag, 2011.

[16] L. G. Kroon, G. Maróti, and L. Nielsen, "Rescheduling of railway rolling stock with dynamic passenger flows," Transp. Sci., pp. 1-20. http://dx.doi.org/10.1287/trsc. 2013.0502

[17] M. Lohatepanont and C. Barnhart, "Airline schedule planning: Integrated models and algorithms for schedule design and fleet assignment," Transp. Sci., vol. 38, no. 1, pp. 19-32, Feb. 2004.
[18] A. Mercier, J. F. Cordeau, and F. Soumis, "A computational study of Benders decomposition for the integrated aircraft routing and crew scheduling problem," Comput. Oper. Res., vol. 32, no. 6, pp. 1451-1476, Jun. 2005.

[19] J. A. Mesa, F. A. Ortega, and M. A. Pozo, "A geometric model for an effective rescheduling after reducing service in public transportation systems," Comput. Oper. Res., vol. 40, no. 3, pp. 737-746, Mar. 2011.

[20] N. Papadakos, "Integrated airline scheduling," Comput. Oper. Res., vol. 36, no. 1, pp. 176-195, Jan. 2009.

[21] J. Pita, C. Barnhart, and A. P. Antunes, "Integrated flight scheduling and fleet assignment under airport congestion," Transp. Sci., vol. 47, no. 4, pp. 477-492, Nov. 2013.

[22] C. G. Walker, J. N. Snowdon, and D. N. Ryan, "Simultaneous disruption recovery of a train timetable and crew roster in real time," Comput. Oper. Res., vol. 32, no. 8, pp. 2077-2094, Aug. 2005.

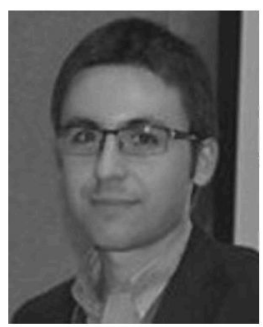

Luis Cadarso was born in 1985. He received the Ph.D. degree in aerospace engineering from the Technical University of Madrid, Madrid, Spain. In September 2013, he joined Rey Juan Carlos University, Madrid, as an Assistant Professor. His research interests include operations research and its application to transportation.

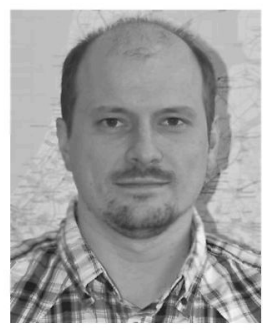

Gábor Maróti was born in 1976 . He received the Ph.D. degree in operations research from the Eindhoven University of Technology, Eindhoven, The Netherlands. He is a Senior Researcher at Netherlands Railways, Utrecht, The Netherlands, and a part-time Assistant Professor at VU University Amsterdam, Amsterdam, The Netherlands. His research focuses on the applications of combinatorial optimization and linear (integer) programming for transportation problems.

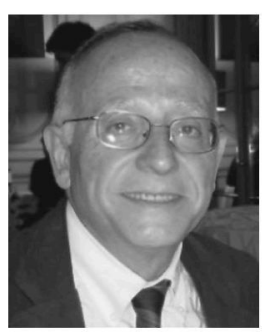

Ángel Marín was born in 1947. He received the Ph.D. degree in aeronautical engineering from the Technical University of Madrid, Madrid, Spain. He has Full Professor position at the Technical University of Madrid. His research activity is focused on problems of network design, large systems, and transportation using optimization methods. 